\title{
VARIATIONS AUTOUR DE LA COMMUNICATION
}

\begin{abstract}
Marc Lits ${ }^{1}$
Les revues scientifiques jouent un rôle déterminant dans la constitution des domaines au sein desquels la recherche s'organise. Comme beaucoup d'autres, les sciences de l'information et de la communication ont utilisé ce vecteur avec un double objectif : se positionner dans le champ scientifique, en tant que jeune discipline émergente, et délimiter les contours théoriques et méthodologiques de la discipline par rapport aux autres sciences humaines.

Point n'est besoin de rappeler que la science est aussi un champ où se confrontent des acteurs en concurrence, où les positions se conquièrent certainement au nom d'une excellence scientifique, mais aussi au prix de combats épistémologiques et politiques. Face à des disciplines bien installées au sein des sciences humaines comme la sociologie, les sciences politiques, la linguistique, voire la sémiologie, les sciences de l'information et de la communication ont dû s'imposer progressivement, en s'appuyant sur des disciplines proches, et en revendiquant progressivement leur autonomie. À l'occasion des cinquante ans d'existence de notre département, Recherches en communication avait tenté de dresser un panorama de ce parcours commencé immédiatement après la
\end{abstract}

1 Marc Lits est professeur au Département de communication de l'UCL, directeur des revues Recherches en communication et Médiatiques, et membre de la rédaction en chef de la revue Hermès.

Recherches en communication, $\mathrm{n}^{\circ} 28$ (2007). 
dernière guerre, et pas encore achevé aujourd'hui ${ }^{1}$. D'autres revues s'y sont également essayées, comme la revue Réseaux à l'occasion de son centième numéro ${ }^{2}$ ou la revue Hermès ${ }^{3}$.

Ces différents numéros commémoratifs ou récapitulatifs ont rencontré les objectifs fixés. Ils ont réussi à montrer comment une discipline nouvelle se construit progressivement en reprenant des éléments à des domaines proches, et comment le montage original de ces fragments importés, leur mise en réseau permettent d'identifier peu à peu un nouveau champ, qui doit bien sûr beaucoup à ses disciplines de référence, mais qui s'est progressivement autonomisé. Il en va des disciplines scientifiques comme de l'histoire des langues. Le français, l'espagnol, l'italien sont bien sûr issus, comme langues romanes, du latin, mais à un moment, ces nouvelles langues sont clairement identifiées par les linguistes comme des langues différentes. Si les Serments de Strasbourg, en 842, ne sont plus considérés comme un texte latin, mais le premier texte écrit en ancien français, c'est parce qu'il utilise des mots légèrement différents du latin, mais surtout parce que sa syntaxe est devenue radicalement autre que celle du latin. La conjugaison, le recours aux cas pour marquer la fonction, la place des mots dans la phrase : autant de traits spécifiques.

Il en va de même pour les sciences de l'information et de la communication. Elles ne peuvent nier leur filiation avec la sociologie, la linguistique, la science politique, la psychologie sociale, mais l'assemblage qu'elles proposent de ces concepts empruntés, leur application à des objets nouveaux (les mass médias principalement) ont déplacé les lignes de frontière. Une autre discipline est là, dans le champ, en gestation progressive, dans une autonomie grandissante, parfois en tension avec ses grandes sœurs (ou ses grand-mères), parfois en dialogue.

Cette histoire, cet héritage, il ne faut pas les nier, les occulter, puisque ces emprunts, ces échanges, ces métissages sont constitutifs de notre discipline. Dès lors, il est intéressant de laisser s'exprimer ces diversités méthodologiques, transdisciplinaires, cette prise en compte d'objets variés. Habituellement, les dossiers des revues sont portés par une équipe, autour d'un axe central fédérateur, qu'il soit de type méthodologique, ou fondé sur des approches complémentaires d'un même

1 Recherches en communication, $\mathrm{n}^{\circ} 11,1999$, « Un demi-siècle d'études en communication ».

2 Réseaux, $\mathrm{n}^{\circ}$ 100, 2000, «Communiquer à l'heure des réseaux ».

3 Hermès, $n^{\circ}$ 48, 2007, « Racines oubliées des sciences de la communication ». 
objet. Et en fin de numéro, quelques varias sont d'habitude destinés à accepter des recherches plus limitées, des études particulières. Ce numéro se veut différent de la logique commune adoptée par notre revue, puisqu'il s'ouvre entièrement à des varias qui ne répondent pas à une commande centrale, qu'il laisse la parole libre à des chercheurs d'horizons différents, qui ne se connaissent pas, qui n'ont jamais travaillé ensemble. Mais dans cet éclatement volontaire, il y a cependant comme une cartographie du champ qui se dessine en creux. À travers les objets retenus, les disciplines de référence, les méthodologies privilégiées, il serait illusoire de chercher une ligne commune, et inexact. Car cela consisterait à ramener de la centralité là où la discipline, précisément, se revendique (c'est en tout cas notre hypothèse et notre conviction) de l'éclatement, du bricolage cher à Michel de Certeau. Cette diversité n'est pas un signe de faiblesse du domaine, c'est au contraire sa spécificité, ce qui fait sa richesse épistémologique. Il serait dangereux de l'oublier, et de vouloir fonder une nouvelle discipline qui renierait ses origines, qui occulterait les apports des disciplines voisines.

Les différents articles repris dans ces variations sont significatifs de cette ouverture du champ. Ils se revendiquent de proximités disciplinaires diverses, mais aussi de méthodologies complémentaires : certains s'appliquent à décrire rigoureusement un objet particulier quand d'autres proposent des réflexions conceptuelles; d'aucuns vont sur le terrain en observateurs nourris de méthodes anthropologiques quand leurs collègues font de l'analyse expérimentale. Il fallait cependant mettre ces articles dans un certain ordre, lequel n'est pas dénué non plus de priorités épistémologiques. Nous commencerons donc par des articles plus réflexifs, proches de la philosophie politique, pour reconnaître que les analyses de l'espace public et l'intérêt pour la communication politique constituent probablement un des socles communs d'une discipline qui a connu ses premiers pas quand des chercheurs américains s'intéressaient aux comportements électoraux et tentaient de trouver les outils permettant de les prédire, pour mieux arriver à les influencer dans les campagnes électorales. Eric Dacheux revient sur un concept abondamment discuté dans nos départements universitaires : l'espace public, véritable pont aux ânes des études en communication. Par contre, Katrien Pype revisite aussi l'espace public, mais en observant comment se construit désormais une sphère publique culturelle, en partie construite par les images télévisuelles, ainsi qu'elle a pu l'observer, avec un regard anthropologique, dans la gigantesque métropole congolaise. Quant à Nicolas Baygert, il repart des théories de l'agenda 
setting et du two-step flow pour analyser la campagne présidentielle de Nicolas Sarkozy en 2007. En anglais pour ces deux derniers articles, puisque notre revue a fait le choix de publier les articles dans les deux langues, français et anglais.

Par contre, Marie-Joseph Bertini choisit de s'intéresser à un apport plus récent aux sciences de l'information et de la communication, celui des cultural studies, qui permettent de regarder autrement nos objets d'étude. Mais si les méthodes évoluent, les objets également, tributaires des transformations sociales. C'est ainsi que Annie-Hélène Le Cornec Ubertini s'intéresse à l'éco-citoyenneté. Ecologie, responsabilité sociale des entreprises, approches renouvelées du marketing : les nouvelles formes du vivre ensemble, les nouveaux modes de consommation sont autant de terrains pris en compte par la psychologie sociale ou la communication d'organisation.

Mais d'autres chercheurs vont au-delà des analyses de cas ponctuelles pour réfléchir aux changements plus profonds induits par les nouveaux dispositifs de communication et les révolutions techniques. Marc Lits regarde ainsi comment les structures de mise en récit sont modifiées par l'usage de nouvelles technologies dans les rédactions. Max Poty montre comment les médias changent aussi notre manière de saisir les événements. Quant à Anne-Catherine Simon et Baptiste Hupin, c'est à la radio qu'ils s'intéressent, et au rôle de la voix dans la transmission de l'information.

On ne pouvait ignorer les médias dans la diversité des études ici rassemblées, et les derniers articles y reviennent, puisque Arnaud Lucien analyse la fonction symbolique du juge dans des documentaires judiciaires, et que Nelly Quemener décrit les différents types d'humour dans des talk shows de la télévision française. Tout est toujours question d'image, ce que démontre finalement Céline Bryon-Portet, à travers les représentations des pilotes de chasse, utilisées à des fins de recrutement par l'armée française. Le mythe n'est jamais loin de la récupération, c'est ce qu'aurait pu dire un sémiologue célèbre, présent dans notre communauté scientifique dans les premiers temps de la création des sciences de l'information et de la communication.

Ainsi, au long de ces articles, dans leurs variations, leur hétérogénéité, se dessine un paysage possible de notre discipline. Que d'autres choix auraient pu faire apparaître bien différent. Heureusement ! 